International Journal of Medical Sciences

ISSN 1449-1907 www.medsci.org 2007 4(2):115-123

Research Paper

(C) Ivyspring International Publisher. All rights reserved

\title{
The characterisation of mucin in a mature ovarian teratoma occurring in an eight year old patient
}

\author{
Anwar Suleman Mall 1, Marilyn Tyler 1, Zoe Lotz ${ }^{1}$, Alan Davidson ${ }^{1}$, Jerry Rodrigues ${ }^{4}$, George van der \\ Watt ${ }^{3}$, Delawir Kahn ${ }^{1}$, Dhirendra Govender ${ }^{2}$ \\ 1. Departments of Surgery, Groote Schuur and Red Cross Hospitals, University of Cape Town, Cape Town, South Africa \\ 2. Anatomical Pathology, Groote Schuur Hospital, University of Cape Town, Cape Town, South Africa \\ 3. Chemical Pathology, Groote Schuur Hospital, University of Cape Town, Cape Town, South Africa \\ 4. Department of Molecular and Cell Biology, University of Cape Town, Cape Town, South Africa
}

Correspondence to: Anwar Suleman Mall, PhD, Department of Surgery, University of Cape Town, J-Floor, Old Main Building, Groote Schuur Hospital, OBSERVATORY 7925, SOUTH AFRICA. Tel: +27 +21 406-6224 Fax: +27 +21 448-6461 Email: Anwar.Mall@uct.ac.za

Received: 2007.02.16; Accepted: 2007.04.02; Published: 2007.04.10

Introduction: The presence of MUC5AC (M1 antigen) and MUC6 have previously been found in ovarian mucinous cyst. We characterized the mucins in the crude mucus and tissue of a mature ovarian teratoma in an 8 year old girl. Materials and Methods: Mucins were purified from crude mucus by density gradient ultra-centrifugation in $\mathrm{CsCl}$ and analysed by gel-filtration and SDS-PAGE analysis. Mucin identification and expression was by western blotting and immunohistochemistry. Results: Histology showed a tumour with solid and cystic areas, with the cysts lined by colonic and respiratory mucosae. Equal volumes of 'sol' and 'gel' phases of approximately $10.0 \mathrm{ml}$ of crude mucus were obtained. Gel filtration and SDS-PAGE analyses suggested that the mucin was mainly of the large polymeric type which dissociated upon reduction of disulphide bonds with DTT. The colonic and respiratory epithelia predominantly expressed acidic mucin of the sialated and sulphated types respectively. MUC1 and MUC1c were expressed exclusively in respiratory epithelium, MUC2 and some MUC6 (focal) in the colonic tissue and MUC5AC in both tissues. Western blotting confirmed the presence of MUC2, MUC5AC and MUC5B in the secreted gel. Serine, threonine and proline made up the bulk of the amino acids in the sample. Discussion: Ovarian teratoma produced a highly viscous mucus secretion in which the mucin was largely polymeric and of the MUC2, MUC5AC and MUC5B type. The respiratory component of the teratoma expressed MUC1 and MUC1c and the colonic components of the teratoma expressed MUC2 and some MUC6. MUC5AC was expressed in both components.

Key words: Mucus, mucins, ovary, teratoma

\section{INTRODUCTION}

Mucins are a family of high molecular-weight, heavily O-glycosylated glycoproteins that are either secreted [1] or are membrane-bound [2].

The physiochemical and biological properties of secreted mucus are largely conferred by mucins that are responsible for the rheological properties of normal mucus gels that coat and protect the epithelial cells of the internal tracts of the body [1]. The mucin protein core consists of highly glycosylated regions (resistant to proteolysis) and regions shown to be non-glycosylated (susceptible to proteolysis) [3]. Cysteines in these 'naked' regions link mucin monomers by disulphide bridges to form large mucin oligomers of 2-40kDa molecular mass [1, 4-7].

Mucin genes are highly polymorphic due to the presence of long stretches of variable number of tandem repeats (VNTRs) that are heavily glycosylated. Thus far five secreted gel-forming mucins have been reported, four of which (MUC2, MUC5AC, MUC5B and MUC6) are coded for by a cluster of genes on chromosome 11p15 [8]. The mucus that forms a continuous, insoluble adherent gel layer in the stomach and which protects the underlying mucosa from the hostile environment of the lumen consists of MUC5AC and MUC6 [9]. Two mucins, MUC5AC and MUC5B, have been convincingly demonstrated to be the major components of the crude mucus gel lining the respiratory tract [10], whilst an up-regulation of MUC2 has been reported in respiratory disease [11]. MUC2 is the major mucin in the crude mucus gel lining the colonic epithelium [12], the deficiency of which can cause mice to develop colitis in the short term [13] and intestinal carcinogenesis at about 6 months [14]. MUC1 was the first reported membrane-bound mucin, widely expressed by normal glandular epithelial cells and dramatically increased in malignant cells of the breast, ovary and pancreas [15].

Mucin genes are independently regulated and their expression is organ and cell type specific [16]. In 1977 Bara et al [17] showed that ovarian mucinous cysts but not ovarian cysts of other histological types, contained M1 antigens common with those of normal 
gastric mucosa and were localised in the mucous secreting cells of the columnar epithelium. The antigens, borne by molecules of large size, were viscous and fractionated at a density of $1.4 \mathrm{gml}^{-1}$ in a $3.5 \mathrm{M} \mathrm{CsCl}$ density gradient, suggesting they were mucins [17]. The gastric M1 mucin was later found to be encoded by the MUC5AC gene [18]. MUC6 was also reported to be a major component of ovarian cyst fluid from a benign serous cyst adenoma [19].

In this study we have characterised the mucus secreted by the right ovary of an eight year old patient with a bilateral ovarian mature teratoma, using both biochemical and histological techniques.

\section{The Patient}

\section{Clinical Findings}

An eight-year-old female presented with a three-week history of abdominal swelling associated with pain. She was a well-looking child with appropriate weight and height for age. She was slightly pale without peripheral oedema. Examination of the abdomen revealed a huge bosselated mass arising from the pelvis, and filling the right flank. CT scan showed a large mass filling the right hemi-abdomen, extending from the level of the renal vein superiorly and abutting the bladder inferiorly. The mass was non-homogenous with solid and cystic components and areas of calcification.

Blood workup showed a microcytic anaemia (Haemoglobin 8.8g/dl and MCV $67 \mathrm{fl}$ ) and normal renal function. Alpha-fetoprotein and beta-human chorionic gonadotrophin levels were both in the normal range. The total protein was decreased at $58 \mathrm{~g} / 1$ $(60-80 \mathrm{~g} / 1)$ and the albumin markedly decreased at $14 \mathrm{~g} / 1(29-42 \mathrm{~g} / \mathrm{l})$. Protein electrophoresis revealed a markedly raised alpha-2 macroglobulin fraction of $20.4 \mathrm{~g} / \mathrm{l}(4-9 \mathrm{~g} / \mathrm{l})$ and the serum cholesterol was elevated at $6.6 \mathrm{mmol} / 1 \quad(<5 \mathrm{mmol} / \mathrm{l})$. The urine protein:creatinine ratio was elevated at $0.26 \mathrm{~g} / \mathrm{mmol}$ $(<0.02 \mathrm{~g} / \mathrm{mmol})$ and the $24 \mathrm{~h}$ urine protein was quantified at $1.9 \mathrm{~g}(0-0.15 \mathrm{~g} / 24 \mathrm{~h})$, confirming nephrotic syndrome.

At surgery bilateral ovarian teratomas - the left larger than the right - were identified and excised. Normal ovarian tissue was preserved on the right. At follow-up her nephrotic syndrome persisted, and renal biopsy showed a mixed membranous/mesangio-capillary glomerulonephritis. She was commenced on steroid therapy and several months later she continues to have proteinuria.

\section{MATERIALS and METHODS}

\section{Ethics}

The University of Cape Town Research and Ethics Committee provided approval for this study to be carried out, ethics number REC REF 302/2005.

\section{Extraction and isolation of ovarian mucin}

A crude specimen of mucus with a total volume of approximately $8-10 \mathrm{ml}$ in a 'sol' and 'gel' phase was obtained from the patient post-operatively. The mucus was diluted at a 1:3 ratio with ice cold buffer containing $6 \mathrm{M}$ guanidinium chloride $(\mathrm{GuHCl}), 1 \mathrm{mM}$ phenylmethylsulfonyl fluoride (PMSF), 5mM EDTA and $5 \mathrm{mM}$ N-ethylmaliemide (NEM) in $0.1 \mathrm{M}$ Tris buffer, $\mathrm{pH} 6.5[5,20,21]$.

Solubilisation of the mucus was by gentle mixing overnight at $4^{\circ} \mathrm{C}$ followed by brief homogenization with an ultra-turrax (Junke and Kunkel, Germany) for $30 \mathrm{~s}$. The solution was centrifuged at $1000 \mathrm{rpm}(6000 \mathrm{~g})$ for $1 \mathrm{~h}$ at $4^{\circ} \mathrm{C}$. The supernatants were strained through glass wool and insoluble material was re-extracted three times and added to the soluble pool. Solubilized glycoprotein was purified in a cesium chloride $(\mathrm{CsCl})$ gradient at a starting density of $1.39 \mathrm{~g} . \mathrm{ml}^{-1}$ to $1.42 \mathrm{~g} \cdot \mathrm{ml}^{-1}$ in $\mathrm{CsCl} / 4 \mathrm{M} \mathrm{GuHCl}[5,20,21]$. Purified mucins were reduced in $6 \mathrm{M} \mathrm{GuHCl}, 5 \mathrm{mM}$ EDTA and $10 \mathrm{~mm}$ dithiothreitol (DTT) in $0.1 \mathrm{M}$ Tris-HCl buffer $\mathrm{pH} 8.0$, or for $5 \mathrm{~h}$ at $37.0^{\circ} \mathrm{C}$ or $0.2 \mathrm{M}$ sodium dihydrogen phosphate buffer, $\mathrm{pH} 8.0$ and subsequently alkylated with $25 \mathrm{mM}$ iodoacetamide (IAA) for $15 \mathrm{~h}$ at room temperature in the dark.

\section{Gel Filtration}

An aliquot of purified mucin and reduced or digested purified mucin was chromatographed on a Sepharose CL-2B column equilibrated and eluted with $0.2 \mathrm{M} \mathrm{NaCl}: 0.02 \%$ sodium azide at flow rate of $40 \mathrm{ml} / \mathrm{h}$ at room temperature. Fractions obtained from both the void and included volumes (proteins) were analyzed by the Periodic Acid Schiffs (PAS) (A $\left.{ }_{555}\right)$ [22] and Lowry $\left(\mathrm{A}_{700}\right)[23]$ assays.

\section{Enzymatic digestion of mucins}

Mucins were digested by adding one volume of the preparation to one volume of 'papain digest buffer' which consisted of papain $(20 \mu \mathrm{g}$ enzyme per $\mathrm{mg}$ of protein to digest) in $0.1 \mathrm{M}$ potassium dihydrogen phosphate/disodium hydrogen phosphate buffer at pH 6.5 containing $0.005 \mathrm{M}$ cysteine and $0.005 \mathrm{M}$ EDTA (final concentration of each reagent). The whole mixture was incubated in a test tube or in a dialysis sac immersed in the digestion buffer (without enzyme) at $60^{\circ} \mathrm{C}$ for $48 \mathrm{~h}$ and then dialysed exhaustively against $0.2 \mathrm{M} \mathrm{NaCl}: 0.02 \%$ sodium azide over 3 changes for 24h.

\section{Polyacrylamide Gel Electrophoresis (SDS-PAGE)}

Mucin samples were prepared in gel loading buffer containing 2\% SDS, $10 \%$ glycerol, and $0.01 \%$ bromophenol blue. 2 ul of $5 \%$ mercaptoethanol was applied to samples and boiled for $2 \mathrm{~min}$. Electrophoresis was performed using a $4 \%$ stacking gel and a $7.5 \%$ running gel with $0.1 \%$ SDS. After electrophoresis gels were stained for carbohydrate with periodic acid/Schiff (PAS) and with Coomassie Brilliant Blue G-250 for protein determining the protein content.

\section{Agarose Gel Electrophoresis and Western Blotting}

Western blot was performed to test for reactivity between the polyclonal antibody and the mucin samples. Samples were electrophoresed in $1.0 \%(\mathrm{w} / \mathrm{v})$ agarose gel prepared in $40 \mathrm{mM}$ Tris-acetate $/ 1 \mathrm{mM}$ 
EDTA, pH 8.0, containing 0.1\% (w/v) SDS. Electrophoresis was performed in a horizontal gel apparatus (90V) using thicker sample combs (12-well, 1.5-mm-thickness) for $2 \mathrm{~h}$ at room temperature. After electrophoresis, mucins were transferred to nitrocellulose membrane by vacuum blotting. After vacuum blotting, the membrane was incubated in phosphate-buffered saline (PBS) with $0.05 \%$ Tween-20 (T-PBS) containing 5\% fat-free dry milk to prevent non-specific binding prior to incubation with the primary antibody. The membrane was then washed with TBST three times for $5 \mathrm{~min}$ and incubated overnight at $4^{\circ} \mathrm{C}$ with primary antibody (MUC2, MUC5AC, MUC5B, (kindly provided by Professor Dallas Swallow, University College, London, UK), diluted in 5\% $(\mathrm{m} / \mathrm{v})$ low fat milk powder in TBST at 1:2000 dilution for MUC5AC and MUC5B, and 1:5000 dilution for MUC2. The membrane was washed 3 times for $5 \mathrm{~min}$ with TBST and incubated for $1 \mathrm{~h}$ with HRPO-conjugated secondary antibody (goat anti rabbit) diluted in $5 \%(\mathrm{~m} / \mathrm{v})$ low fat milk powder in TBST at 1:5000 dilution. The membranes were then washed three times with TBST. Bands that supported the binding of the antibody to the mucin were visualized by using the ECL detection kit.

\section{Analytical determinations}

Glycoprotein was estimated by the PAS procedure [22], protein according to the method of Lowry [23].

\section{Amino acid analysis}

Amino acid analyses were performed in the Department of Molecular and Cell Biology, University of Cape Town. The amino acid content of purified freeze-dried mucins were analysed using a high pressure liquid chromatography (HPLC) system. The mucin samples were hydrolysed in the gas-phase using a similar method to that reported by Cohen et al [24] and the analysis procedure followed was that of Klapper [25]. Briefly, the samples were vacuum dried and hydrolysed by constant boiling $\mathrm{HCl}$ and $1 \%(\mathrm{w} / \mathrm{v})$ phenol. The vessels were purged with nitrogen gas and sealed under vacuum followed by hydrolysis in the gas phase at $110^{\circ} \mathrm{C}$ for $24 \mathrm{~h}$. Following hydrolysis, the vials were cooled and vacuum dried to remove the residual $\mathrm{HCl}$. The dried samples were redissolved in citrate buffer $\mathrm{pH} 2.2$ and injected into a HPLC column from Waters Associates, Medford, MA., packed with a cation exchange resin (sulfonated polystyrene crosslinked with divinylbenzene) and eluted with a series of buffers ranging from a low $(0.20 \mathrm{M}$ sodium citrate, $\mathrm{pH} 3.05)$ to high $(0.25 \mathrm{M}$ sodium nitrate, $0.04 \mathrm{M}$ borate $\mathrm{pH}$ 9.5) $\mathrm{pH}$. Detection was carried out using post-column derivatization with o-phthalaldehyde (OPA), a fluorescent reagent that reacts with all the amino acids except proline. Proline analysis was carried out in the same instrument except that the eluting amino acids were reacted with sodium hypochlorite before detection with the OPA reagent. The relative ratios of the individual amino acids for each sample was determined and compared to each other.

\section{Specimen processing and histochemistry}

All specimens were fixed in $10 \%$ buffered formalin and embedded in paraffin wax. The sections were stained routinely with haematoxylin and eosin (H\&E). Selected sections were stained with high iron diamine (HID)/Alcian blue and periodic acid Schiff (PAS)/Alcian blue.

\section{Immunohistochemistry}

Monoclonal antibodies to MUC1, MUC1core (MUC1c), MUC2, MUC5AC and MUC6 were bought from Novacastra Laboratories (Newcastle-Upon-Tyne, UK). The antibody to MUC5B was from Santa Cruz Laboratories. Secondary antibodies, Envision labeled polymer-HRP anti mouse antibody and monoclonal antibody (Clone 11-7) to CEA were bought from DakoCytomation. Paraffin embedded tissue blocks were obtained from the archives of the Division of Paediatric Pathology (Anatomical Pathology) at the Red Cross Children's hospital.

Paraffin sections were fixed onto APES coated slides overnight in an incubator at $50-55^{\circ} \mathrm{C}$. Sections were dewaxed in xylol and rehydrated through descending graded alcohols to distilled water. Sections were incubated in $1 \% \mathrm{H}_{2} \mathrm{O}_{2}$ methanol for $15 \mathrm{~min}$ to block endogenous peroxidase activity. Sections were washed in running tap water. Heat induced antigen retrieval was achieved by pressure cooking in a $0.01 \mathrm{M}$ citrate buffer $(\mathrm{pH} 6)$ for $2 \mathrm{~min}$ and cooled. Sections were washed in three changes of PBST for $5 \mathrm{~min}$ each. Non-specific antibody-binding was blocked by incubating the sections with $10 \%$ normal goat serum for $10 \mathrm{~min}$. Sections were incubated with primary mouse antibody (MUC1, MUC1core, MUC2, MUC5AC, MUC6 and CEA) for 30min, except for MUC2 which was for $60 \mathrm{~min}$ at room temperature. Sections were washed again in three changes of PBST for $5 \mathrm{~min}$ each and then incubated with EnVision anti-mouse (Dakocytomation EnVision + system labeled polymer-HRP anti-mouse) for 30min. Sections were washed in PBST and colour developed using chromogen (DAB) for $10 \mathrm{~min}$. Sections were then rinsed with PBST, water and then colour enhanced in 1\% copper sulphate for $10 \mathrm{~min}$. Finally, sections were counterstained in Mayer's haematoxylin, dehydrated through the ascending graded alcohols, cleared in xylol and finally coverslipped using Entellan.

\section{RESULTS}

\section{Pathological findings}

The results of laboratory investigations which were out of the normal reference ranges were as follows: total protein $58 \mathrm{~g} / 1(60-80 \mathrm{~g} / \mathrm{l})$, albumin $14 \mathrm{~g} / 1$ $(29-42 \mathrm{~g} / \mathrm{l})$, alpha-2 macroglobulin 20.4g/1 (4-9g/1), cholesterol $6.6 \mathrm{mmol} / 1 \quad(<5 \mathrm{mmol} / 1)$, urine protein:creatinine ratio $0.26 \mathrm{~g} / \mathrm{mmol}(<0.02 \mathrm{~g} / \mathrm{mmol}), 24 \mathrm{~h}$ urine protein of $1.9 \mathrm{~g}(0-0.15 \mathrm{~g} / 24 \mathrm{~h})$. A renal biopsy showed a mixed membranous / mesangio-capillary glomerulonephritis. 


\section{Macroscopy}

The specimen consisted of two pieces of tissue measuring $80 \times 60 \times 50 \mathrm{~mm}$ and $115 \times 70 \times 75 \mathrm{~mm}$. The smaller specimen appeared firm and lobulated on cut section. The larger specimen was solid and multi-cystic with the largest cyst measuring $70 \mathrm{~mm}$ in greatest dimension.
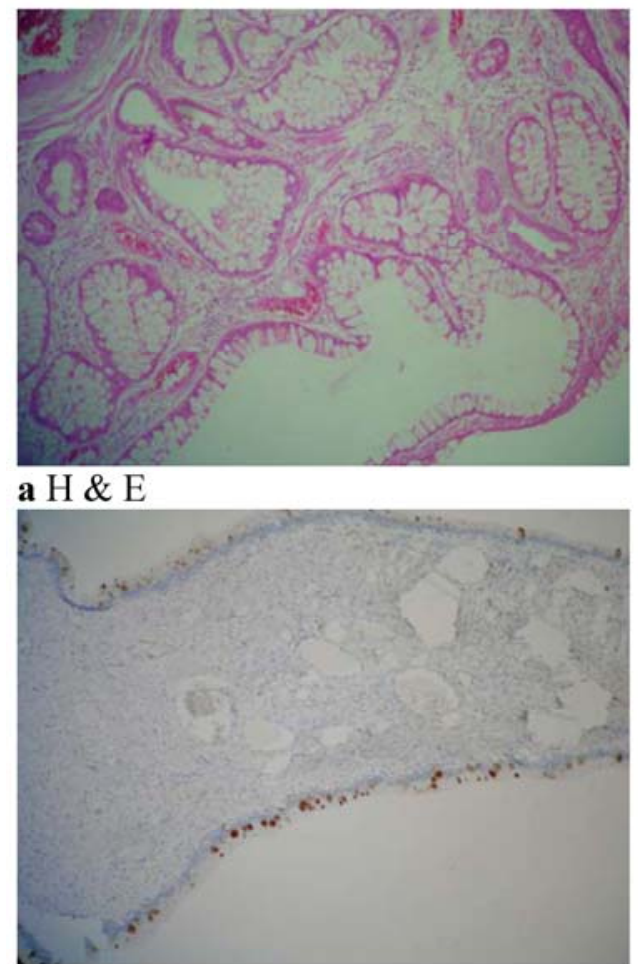

\section{c MUC1}

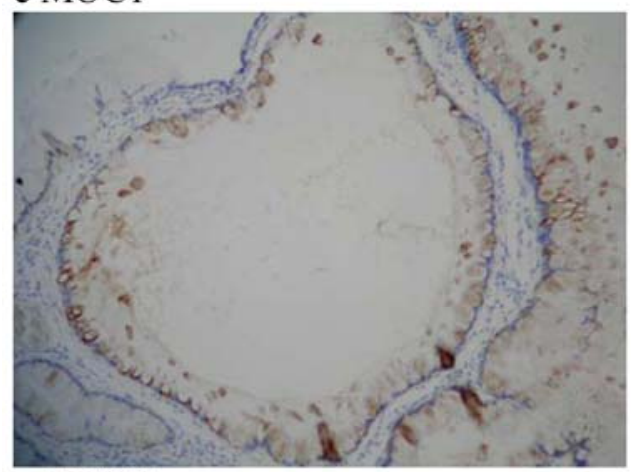

e MUC2

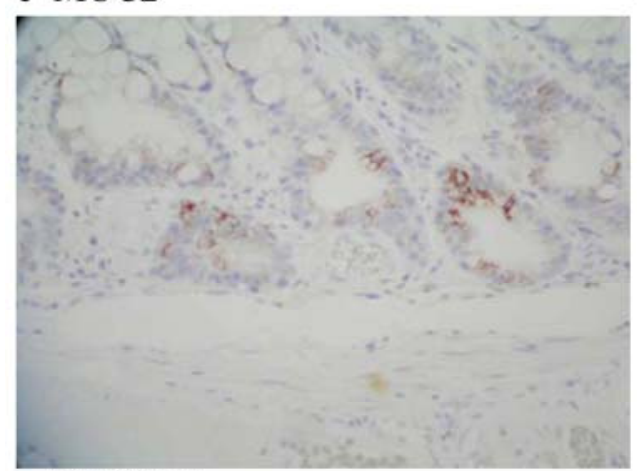

g MUC5AC

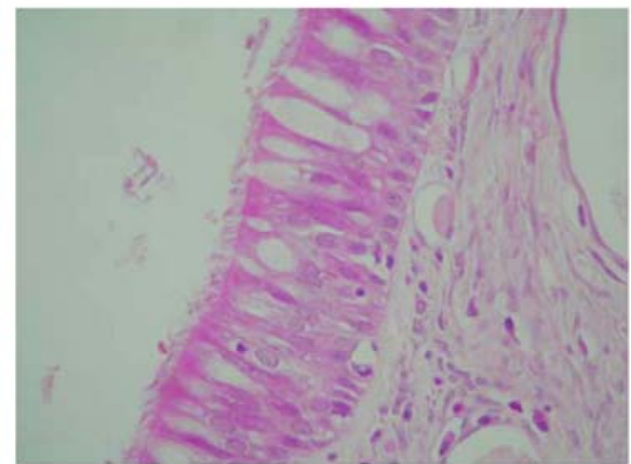

b $\mathrm{H} \& \mathrm{E}$

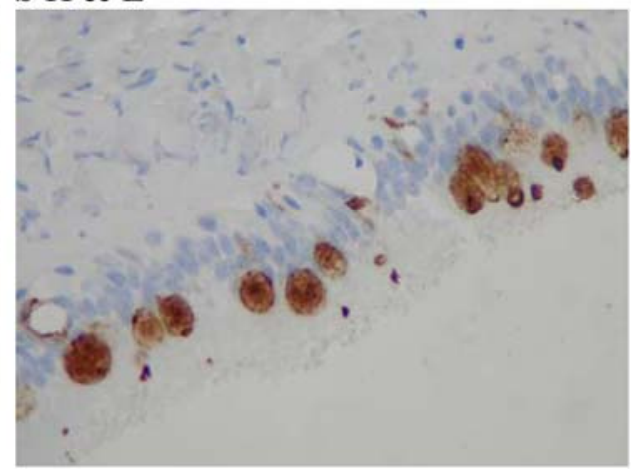

d MUC1

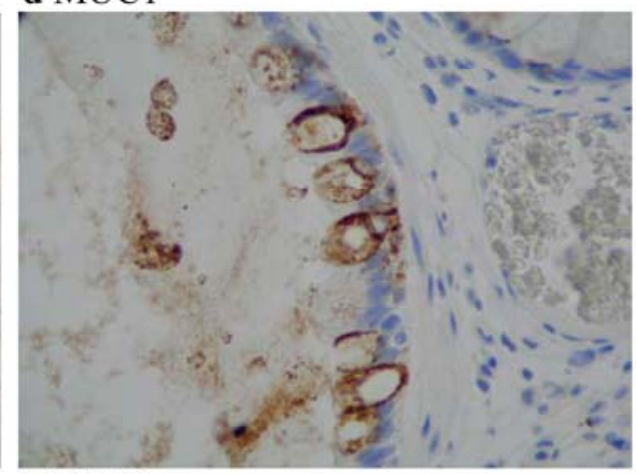

f MUC2

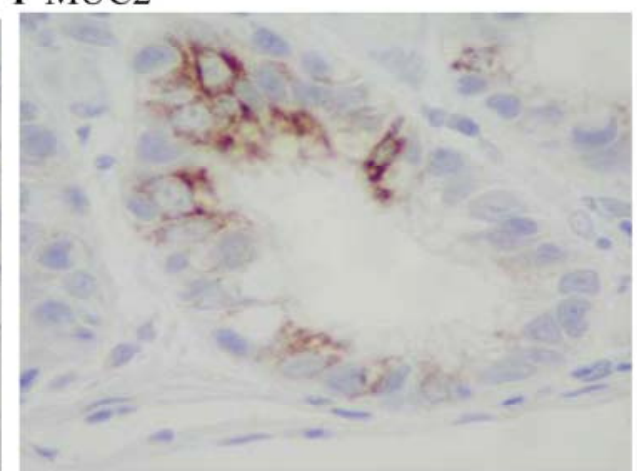

h MUC5AC

\section{Microscopy}

Histologic sections of both specimens showed tumours with solid and cystic areas. The cysts were lined by variable mucosae, including colonic type (Fig. 1a) and respiratory type mucosae (Fig. 1b). Deep to the cyst epithelial lining was a layer of smooth muscle of variable thickness. One cyst was lined by stratified squamous epithelium and contained laminated keratin. No viable skin adnexal structures were noted. A significant part of the solid component of the tumour was composed of blood vessels including many large cavernous vascular spaces. The blood vessels were separated by loose myxoid stroma and smooth muscle. The vessels were lined by bland flattened endothelial cells. Some vessels contained organising fibrin thrombi while others showed concentric hyalinisation and luminal narrowing. In addition, there was prominent degenerative change consisting of calcification, foam cells, cholesterol clefts and haemosiderin deposits. There was no evidence of immature or malignant tissue. The histological features were those of a benign cystic teratoma.

Figure 1. Histology of a mature ovarian teratoma. Colonic type mucosa lining a cyst within the mature cystic teratoma $(\mathrm{H} \& \mathrm{E}$, 40X) (a) and respiratory type epithelium-pseudostratified ciliated columnar epithelium $(\mathrm{H} \& \mathrm{E}$, 160X) (b). MUC1 immunohistochemistry of respiratory epithelium showing cytoplasmic staining of goblet cells (40X) (c) and (160X) (d). MUC2 immunohistochemistry of colonic crypt epithelium showing expression (80X) (e) and (160X) (f). Immunohistochemical expression of MUC5AC in colonic type epithelium (80X) $(\mathrm{g})$ and $(160 \mathrm{X})$ (h). 
Table 1. Mucin histochemistry and immunohistochemistry. Paraffin embedded blocks were retrieved from the Department of Pathology Red Cross Hospital together with the report of a paediatric pathologist. The results were compiled by a pathologist Prof Govender (DG) Anatomical Pathology Groote Schuur Hospital, South Africa. Grading: $0=$ neg; $-1=<5 ; 1=5-25 \% ; 2=26-50 \%$; $3=51-75 \%$ and $4=>75 \%$

\begin{tabular}{|c|c|c|c|c|c|c|c|}
\hline H\&E & PAS/AB & HID & MUC1 & $\begin{array}{c}\text { MUC1 } \\
\text { Core }\end{array}$ & MUC2 & MUC5AC & MUC6 \\
\hline $\begin{array}{c}\text { Colonic mucous } \\
\text { lining }\end{array}$ & Colon PRED.AB & Almost exclusively AB POS & NEG & NEG & $\begin{array}{c}\text { Colon } \\
2+\end{array}$ & $1+$ & Focal \\
$1+$
\end{tabular}

\section{Histology, Histochemistry and Immunohistochem- istry}

Table 1 is a summary of the histochemistry and immunohistochemistry of the ovarian cyst tissue. The tissue showed elements of both colonic and respiratory epithelium (Fig. 1a and b). Whilst both elements had neutral and acidic mucin, the respiratory element had more sulphated acidic than sialated mucin, whilst the colonic epithelium showed no mucin of the sulphated type, there being Alcian Blue sialated mucin only.

The respiratory epithelium was positive for MUC1 (Fig. 1c and d) (26-50\%) and MUC1c. MUC2 was expressed in the colon (Fig. 1e and $\mathrm{f}$ ) and MUC5AC in both colonic (Fig. 1g and h) and respiratory type epithelia. Focal MUC6 was seen in the colonic epithelium. None of the tissue was positive for MUC5B. The colonic epithelium also expressed carcinoembryonic antigen (CEA) but there was no CEA expression in the renal glomeruli.

\section{Purification of mucins by $\mathrm{CsCl}$ density gradient ultra-centrifugation}

Teratomatous mucins were further purified by density gradient centrifugation, twice in $\mathrm{CsCl} / 4 \mathrm{M}$ GuHCl with a buoyant density between 1.39 and $1.40 \mathrm{~g} / \mathrm{ml}$ to remove proteins and nucleic acids. The purification profile in Figure 2, after the second spin demonstrates a clear separation of the lower density proteins positive for Lowry from the higher-density glycoproteins positive for PAS. The mucin-rich fractions were pooled, dialysed against three changes of distilled water and lyophilized.

\section{Gel filtration}

On gel filtration on Sepharose 2B the purified ovarian cyst sample contained both excluded $\left(\mathrm{V}_{\mathrm{o}}\right)$ glycoprotein (indicative of high molecular weight gel-forming native glycoprotein) $[6,26,27]$ and some lower molecular weight glycoprotein as shown by the peak tapering into the included volume $\left(\mathrm{V}_{\mathrm{i}}\right)$ of the column (Fig. 3a). More low molecular weight included volume of the material was seen for mucin treated with DTT which reduced disulphide bonds and digestion with papain resulted in all of the mucin eluting in the included volume of the column (Fig. 3b). This behaviour has been shown for mucin isolated from crude gels of other sources, for example gastric mucus $[3,6,27]$.

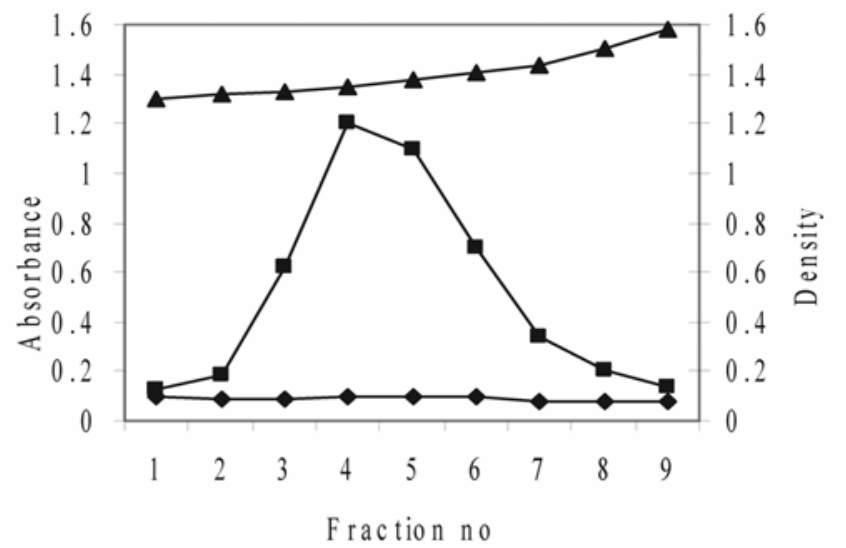

Figure 2 The purification of mucins after a second centrifugation step in a $\mathrm{CsCl}$ density gradient. Solid $\mathrm{CsCl}$ was added to semi-purified mucins obtained from the first density centrifugation spin to give a starting density of $1.39-1.40 \mathrm{~g} / \mathrm{ml}$. After centrifugation $(40,000 \mathrm{rpm}$ for $48 \mathrm{~h})$ the tubes were fractionated into 8 equal fractions and the density of each fraction measured $(\boldsymbol{\Delta})$. The fractions were then assayed for protein absorbance at $280 \mathrm{~nm}(\bullet)$ and analysed for carbohydrate with PAS at $555 \mathrm{~nm}$ (घ)

\section{SDS-PAGE}

With equal loading of purified mucin on $4-20 \%$ SDS-PAGE for both Coomassie Blue protein and PAS stains (Fig. 4), large molecular weight mucin was at the top of the stacking gel, indicative of size $220 \mathrm{kDa}$ and above (Fig. 4, lanes 2-5). Treatment with DTT which reduced disulphide bonds released three visible bands which stained for protein (lane 3, arrows) and showed a little more PAS positive carbohydrate material penetrating the gel (lane 5 arrows), compared with untreated purified mucin which had two protein bands and no carbohydrate (PAS positive bands besides the material at the top of the running gel (lanes 2 and 4).

\section{Agarose Gel Electrophoresis and Western Blotting}

Western Blotting showed the presence of MUC2, MUC5AC and MUC5B in the sample (Fig. 5). Each experiment had a positive and negative control, confirming the specificity of the antibody.

\section{Amino Acid Analysis}

The results are shown in Table 2 with the signature amino acids for mucins, namely, serine, threonine and proline comprising $48.3 \%$ of the total amino acids in the sample. The threonine levels in this particular case were $28.6 \%$ of the total amino acids. 


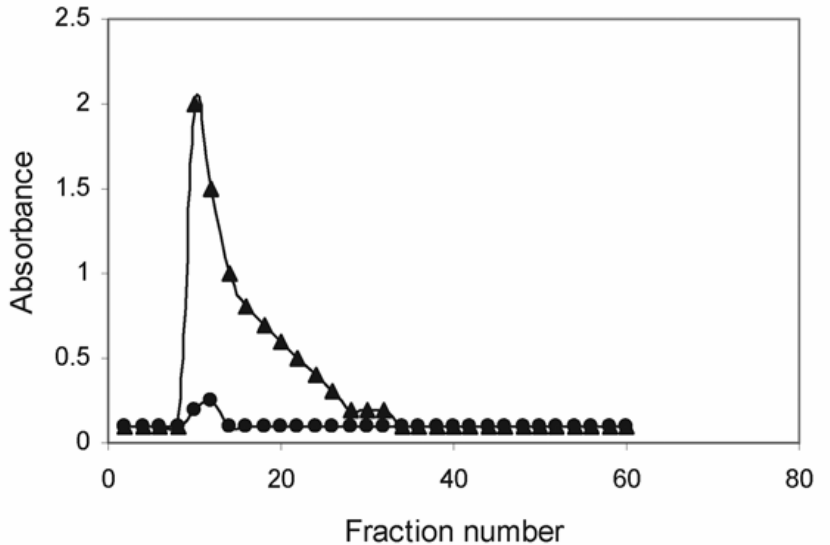

a

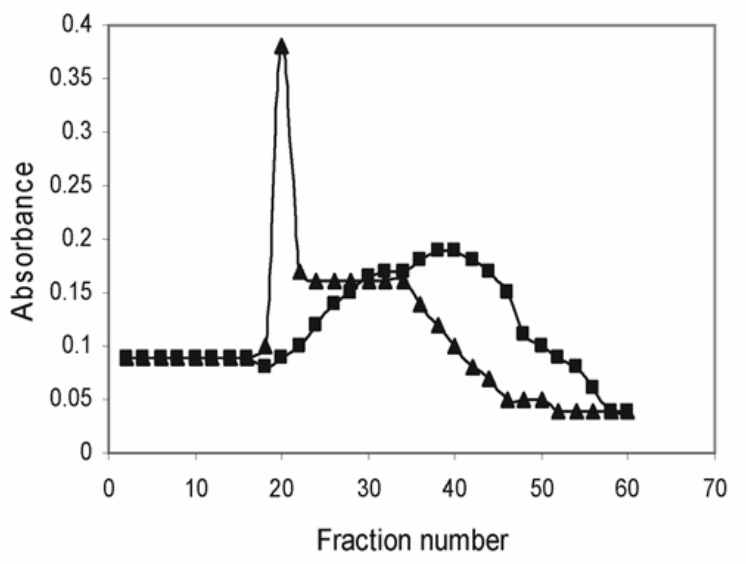

$\mathrm{b}$

Figure 3 Analysis by gel filtration on Sepharose CL-2B of purified mucin obtained from a resected ovarian teratoma specimen. The mucus, extracted in $6 \mathrm{M}$ guanidinium chloride $(\mathrm{GuHCI})$ containing10mM EDTA, 5mM NEM and $1 \mathrm{mM}$ PMSF was purified in a density gradient ultra-centrifugation in $3.5 \mathrm{M} \mathrm{CsCl} / 4 \mathrm{M} \mathrm{GuHCl}$ and chromatographed and eluted with $0.2 \mathrm{M} \mathrm{NaCl}$ : $0.02 \%$ sodium azide at flow rate of $2.0 \mathrm{ml} / \mathrm{min}$ at room temperature Fractions were analysed for carbohydrate with PAS at $555 \mathrm{~nm}(\boldsymbol{\Delta})$ and protein absorbance at $280 \mathrm{~nm}(\bullet)$ (a). Mucin was either reduced with 10mM DTT $(\boldsymbol{\Delta})$ or digested with papain $(\boldsymbol{\bullet})$ before gel chromatography (b).

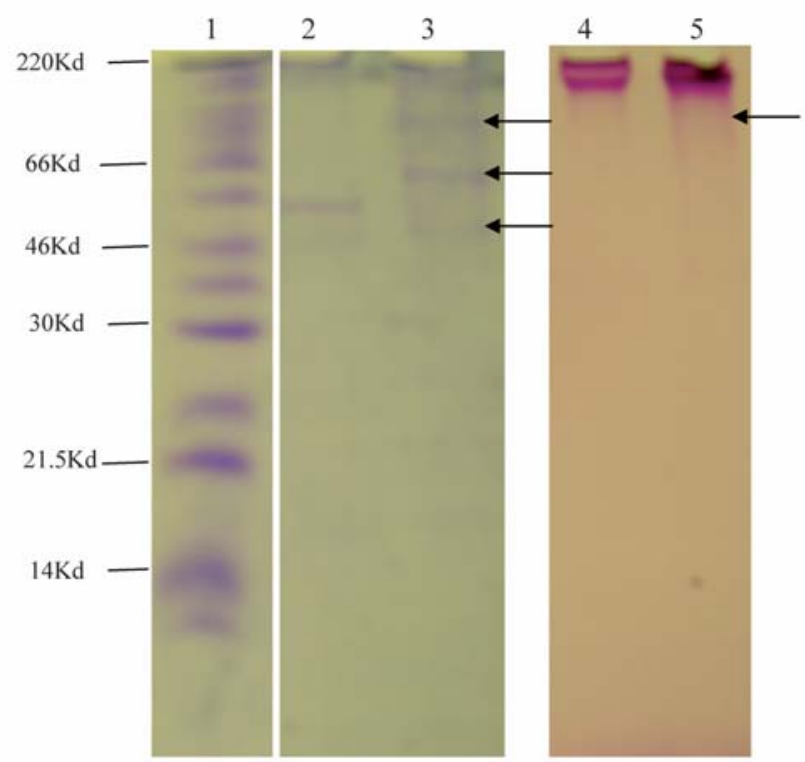

Figure 4 4-20\% gradient SDS-PAGE of purified mucin stained for protein (Lanes 1-3) or carbohydrate (Lanes 4 and 4). Lane 1: Molecular Weight marker Lane 2 and Lane 4. Purified mucin. Lanes 3 and 5, mucin reduced with DTT.

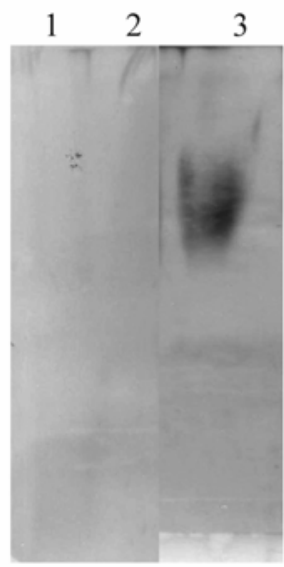

(a)

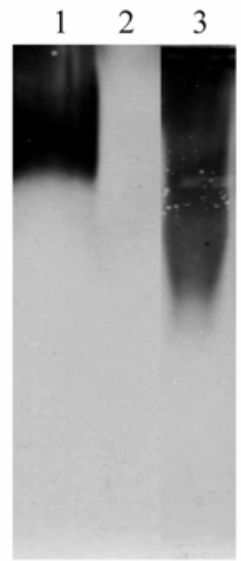

(b)



(c)
Figure 5 Western blotting of purified PMP using rabbit anti-MUC2, MUC5AC and MUC5B polyclonal antibodies. Samples were separated on a $10 \%$ agarose gel and transferred to nitrocellulose membrane by vacuum blotting. The membranes were then incubated with rabbit anti-MUC2 (a), MUC5AC (b) and MUC5B (c) polyclonal antibodies respectively mucins were detected by exposing the membrane to ECL. a) Lanes: $1=$ Cervical (+ve control), 2 Saliva (-ve control) and 3 Ovarian mucus. b) Lanes: 1 Tracheal sputum (+ve control), 2 Normal colon (-ve control), 3 Ovarian mucus. c) Lanes: 1 Tracheal sputum (+ve control), 2 normal colon (-ve control) and 3 ovarian mucus 
Table 2: Amino acid analysis of purified mucin from a mature ovarian cyst teratoma

\begin{tabular}{|c|c|}
\hline Amino acid & Mole \% \\
\hline Aspartic acid (D) & 4.1 \\
\hline Threonine (T) & 28.6 \\
\hline Serine (S) & 8.4 \\
\hline Glutamic acid (E) & 7.0 \\
\hline Proline (P) & 11.3 \\
\hline Glycine (G) & 5.3 \\
Alanine (A) & 3.7 \\
\hline Cysteine (C) & 1.6 \\
Valine (V) & 4.5 \\
\hline Isoleucine (I) & 2.9 \\
\hline Leucine (L) & 3.3 \\
Tyrosine (Y) & 1.2 \\
\hline Phenylalanine (F) & 1.6 \\
\hline Histidine (H) & 12.9 \\
\hline Lysine (K) & 1.9 \\
Arginine (R) & 1.7 \\
\hline
\end{tabular}

\section{DISCUSSION}

Early work by Bara et al (for example ref [17]) showed that the gastric mucin M1 antigens reacted to monoclonal antibodies obtained against mucins isolated from a human ovarian mucinous cyst. These monoclonal antibodies exclusively stained the surface gastric epithelium of normal human gastro-intestinal tract and reacted with fetal, precancerous and cancerous colonic mucosa but not with normal colon [17]. The antibodies also reacted against mucins purified from gastric tissue of patients with intestinal, diffuse and mixed adenocarcinomas [27]. These M1 antigens are encoded by the MUC5AC gene [18].

The 'gel' phase of the crude secreted mucus (5-6ml) was very viscous as observed upon inversion and required solubilisation both by mixing overnight and brief homogenisation. Caesium chloride density gradient ultracentrifugation is an established procedure to isolate and purify mucins from crude mucus secretions [28]. The purification process of the mucins in this study and the outcome of their biochemical characterisation by the methods of gel filtration and SDS-PAGE analysis, before and after treatment with DTT and papain, coincide with the long established model of mucin structure [see ref 1]. Mucins are composed of subunits that form polymers through disulphide bonds. Mucin polymers are large and gel-forming [1] and elute in the $V_{o}$ of a Sepharose 2B gel-filtration column (Fig. 3a) or appear at the top of the running gel when subjected to SDS-PAGE analysis (Fig. 4). Reduction with DTT increased the mobility (Fig. 4) on gels and elution into the included volume of the column as so did digestion with papain (Fig. 3b). The sample had a mixture of polymer and subunit as seen by the profiles (Fig. 3a and b) [26, 27] and whilst reduction was not quite complete, digestion resulted in most of the mucin eluting in the included volume of the column, suggesting the findings of previous studies that the mucin sample could have had a fraction resistant to reduction [12]. The viscous nature of the secretion suggested that it would contain gel-forming mucins and this, to a great extent guided our choice of antibodies in this study.

Histology showed mature cystic teratomas containing cysts lined by both colonic and respiratory type epithelia. Therefore the Western blot analysis showing MUC2 (colon), MUC5AC and MUC5B (respiratory tract) was no surprise. The expression of MUC1 and MUC1c in the respiratory epithelium of the tissue raises interesting questions. MUC1 has long been known to be a prognostic factor in various cancers and is highly expressed in cancers of the breast, ovary, pancreas and colon [15, 29-31]. We have also shown the presence of some MUC5AC in the tissue as reported by Bara et al [17], and MUC6 which was found to be a major component of ovarian cyst fluid of a benign serous cystadenoma [18]. MUC5AC has previously been shown in mucinous carcinomas of the colon [32] and its absence in tumours may be a prognostic factor for more aggressive colorectal carcinoma [33]. There was also a focal expression of MUC6 in the colonic epithelium in the teratoma (Table 1), a finding we have also made in a study on colorectal carcinoma [34]. The tissue showed mucin of predominantly the acidic type, the sulphated type dominating in the respiratory epithelium. Acidic mucins especially the sulphated type, over the PAS positive neutral mucin is a feature of various cancers including cancer of the colon [33]. The amino acid analysis of the purified mucin gave high amounts of 'mucin-like amino acids, serine, threonine and proline. Serine and threonine are points of O-glycosylation found in the tandem repeat regions of mucins and their ratios can vary with the site of secretion and whether the organ is in a normal or diseased state. As far as we know this is the first time an amino acid analysis has been done of purified mucin in an ovarian teratoma.

The observation that this ovarian teratoma produced a highly viscous mucus secretion in which the mucin was largely polymeric and of the MUC2, MUC5AC and MUC5B type is an important one. The respiratory component of the teratoma expressed MUC1 and MUC1c and the colonic components of the teratoma expressed MUC2 and some MUC6 whilst MUC5AC was expressed in both components. Previous studies have shown that secreted mucins such as MUC2 in gels of the small intestine, even after extensive treatment with guanidinium chloride gave an insoluble glycoprotein complex, suggesting that there could be a greater involvement of covalent bonds in the formation of small intestinal mucus, quite resistant to reduction or digestion [12]. MUC5B, when secreted as two glycoforms in the respiratory mucus of a patient who died in status asthmaticus, formed a viscid exudate that was resistant to solubilisation and sequential extraction even in 6M guanidinium chloride 1995 [35].

This patient also had nephrotic syndrome as a result of a mixed membranous / mesangio-capillary glomerulonephritis. Nephrotic syndrome is a well described paraneoplastic phenomenon which usually 
resolves with treatment of the primary tumour. It has been linked to malignant ovarian tumours, both carcinomas [36, 37] and germ cell tumours [38]. There is only one case of nephrotic syndrome associated with a benign ovarian tumour in the literature. A seven year-old girl presented with steroid resistant nephropathy on the basis of membranous glomerulonephritis which resolved completely within two weeks of surgical excision of the tumour [39]. The fact that our patient's nephrotic syndrome was not ameliorated by resection of her tumour, points to two pathologies rather than a paraneoplastic phenomenon. Membranous glomerulornephritis has been reported in a patient with colonic carcinoma. CEA was expressed by the tumour and found in the glomerular deposits [40]. In the case reported here, CEA was expressed in the colonic epithelium in the teratoma but not in the glomeruli.

In summary, cysts in a mature cystic teratoma of the ovary had a viscous gel secretion containing MUC2, MUC5AC and MUC5B with considerably more polymer to degraded subunit which was resistant to reduction of disulphide bonds with DTT and completely digestible with papain. The tissue showed colonic and respiratory elements, expressed mainly acidic mucin of the sulphated type, in the form of MUC1 MUC1c, MUC2 and lesser MUC5AC and MUC6.

\section{Abbreviations}

$\mathrm{CsCl}$ : Caesium chloride; $\mathrm{GuHCl}$ : guanidinium chloride; VNTRs: variable number tandem repeats; PI: proteolytic inhibitors; SDS-PAGE: sodium dodecyl sulphate; DTT: dithiothreitol; IAA: Iodoacetamide; MUC1c: MUC1 core; PBST: Phosphate buffered saline with Tween; CEA: carcino-embryonic antigen.

\section{Acknowledgements}

This work was supported by the South African Medical Research Council, and the University of Cape Town Research Fund. Professor Colin Sinclair-Smith of Red Cross Children's Hospital provided the paraffin blocks for histology.

\section{Conflict of interest}

The authors have declared that no conflict of interest exists.

\section{References}

1. Allen A. Structure and Function of Gastrointestinal mucus. In: Johnson LR, ed. Physiology of the Gastrointestinal Tract. New York: Raven Press; 1981: 617-639.

2. Hollingsworth MA, Swanson BJ. Mucins in cancer: protection and control of the cell surface. Nat Rev Cancer. 2004; 4: 45-60.

3. Scawen M, Allen A. The action of proteolytic enzymes on the glycoprotein from pig gastric mucus. Biochem. J. 1977; 163: 363-368.

4. Minkiewicz-Radziejewska I, Gindzienski A, Zwierz K. Disulfide-Bound Proteolytic Fragments of Gastric Mucin are 100- and 140-kDa Proteins. Biochem Biophys Res Comm. 2000; 270: 722-727.

5. Carlstedt I, Lindgren I, Sheehan JK, et al. Isolation and characterisation of human cervical-mucus glycoproteins. Biochem J.
1983; 211: 13-22.

6. Pearson JP, Allen A, Parry S. A 70000 molecular-weight protein isolated from purified pig gastric mucus glycoprotein by reduction of disulphide bridges and its implication in the polymeric structure. Biochem J. 1981; 197: 155-162.

7. Roberton AM, Mantle M, Fahim REF, et al. The putative 'link' glycopeptide associated with mucus glycoproteins. Composition and properties of preparations from the gastrointestinal tracts of several mammals. Biochem J. 1989; 261: 637-647.

8. Rose MC, Voynow JA. Respiratory tract mucin genes and mucin glycoproteins in health and disease. Physiol Rev. 2006; 86: 245-278.

9. Nordman H, Davies JR, Lindell G, et al. Gastric MUC5AC and MUC6 are large oligomeric mucins that differ in size, glycosylation and tissue distribution. Biochem J. 2002; 364: 191-200.

10. Thornton DJ, Gray T, Nettesheim P, et al. Characterization of mucins from cultured normal human tracheobronchial epithelial cells. Am J Physiol Lung Cell Mol Physiol. 2000; 278: L1118-L1128.

11. Voynow JA. What does mucin have to do with lung disease? Paediatr Respir Rev. 2002; 3: 98-103.

12. Herrman A, Davies JR, Lindell G, et al. Studies on the 'insoluble' Glycoprotein Complex from Human Colon. J Biol Chem. 1999; 274: 15828-15836.

13. Van der Sluis $M$, de Koning BA, de Bruin ACJM, et al. Muc2-deficient mice spontaneously develop colitis, indicating that Muc2 is critical for colonic protection. Gastroenterology. 2006; 131: 117-129.

14. Velcich A, Yang W, Heyer J, et al. Colorectal cancer in mice genetically deficient in the mucin Muc2. Science. 2002; 295: 1726-1729.

15. Taylor-Papadimitriou J, Burchell J, Miles DW, et al. MUC1 and Cancer. Biochim Biophys Acta. 1999; 1455: 301-313.

16. Ho SB, Niehans GA, Lyftogt C, et al. Heterogeneity of Mucin Gene Expression in Normal and Neoplastic Tissues. Cancer Res. 1993; 53: 641- 651

17. Bara J, Gautier R, Mouradian P, et al. Oncofetal Mucin M1 epitope family: Characterisation and expression during colonic carcinogenesis. Int J Cancer. 1991; 47: 304-310

18. Bara J, Chastre E Mahiou J, et al. Gastric M1 mucin and early oncofetal marker of colon carcinogenesis, is encoded by the MUC5AC gene. Int J Cancer. 1998; 75: 767-773.

19. Lloyd KO, Yin BW, Tempst P, et al. MUC-6 mucin is a major component of "blood group substance" from human ovarian cyst fluid. Biochim Biophys Acta. 2000; 1474: 410-4.

20. Mall AS, Sellers LA, Allen A. Purification of pig duodenal mucus glycoprotein from protein and nucleic acid. Biochem Soc Trans. 1987; 15: 1047-1048.

21. Mall AS, Hutton DA, Coan RM, et al. Gastric Mucins:different size of subunit according to reducing conditions. Biochem Soc Trans. 1988; 16: 585-586.

22. Mantle M, Allen A. A colorimetric assay for glycoprotein based on the periodic-acid-Schiff stain. Biochem Soc Trans. 1978; 6: 607 -609 .

23. Lowry OH, Rosebrough NJ, Farr AL, et al. Protein measurement with the Folin phenol reagent. J Biol Chem. 1951; 193: 265-275.

24. Cohen A, Tarvin TL, Bidlingmeyer BA. Analysis of amino acids using postcolumn derivatization with phenylisothiocyanate. American laboratory; 1984; 16: 48-59.

25. Klapper DG. A new low cost, fully automated amino acid analyzer. In: Elzinga M, ed. Methods in protein sequence analysis. New Jersey: Humana press Clifton; 1982; 509-517.

26. Younan F, Pearson J, Allen A, et al. Changes in the structure of the mucous gel on the mucosal surface of the stomach in association with peptic ulcer disease. Gastroenterology. 1982; 82: 827-831.

27. Mall AS, Mcleod HA, Hickman R, et al. Fragmentation Pattern 
of Mucins in Normal and diseased gastric mucosae: A Glycoprotein Fractionates with Gastric mucins Purified from mucosal Scrapings of cancer and Peptic Ulcer Patients. Digestion. 1999; 60: 216-226.

28. Creeth JM, Denborough MA. Density gradient equilibrium methods applied to blood-group specific glycoproteins. FEBS Lett. 1970; 6: 117-120.

29. Taylor-Papadimitriou J, Gendler S. Structure, biology and possible clinical application of carcinoma-associated mucins. Int J Oncol. 1992; 1: 9-16.

30. Manne U, Weiss LH, Grizzle WE. Racial differences in the prognostic usefulness of MUC1 and MUC2 in colorectal adenocarcinomas. Clin Cancer Res. 2000; 6: 4017-4025.

31. Ishizu H, Kumagai J, Eishi Y, et al. Mucin core protein expression by colorectal mucinous carcinomas with or without mucus hyperplasia. J Gastroenterol. 2004; 39: 125-132.

32. Kocer B, Soran A, Erdogan S, et al. Expression of MUC5AC in colorectal carcinoma and relationship with prognosis. Pathol Int. 2002; 52: 470-7.

33. Jass JR, Roberton AM. Colorectal mucin histochemistry in health and disease: A critical review. Pathol Int. 1994; 44: 487-504.

34. Chirwa N, Mall A, Tyler M, et al. The biochemical and immunohistochemical characterization of mucins in colonic disease. A pilot study. S Afr J Surg. 2007; 45: 18-23.

35. Sheehan JK, Richardson PS, Fung DCK, et al. Analysis of Respiratory mucus glycoproteins in asthma: a detailed study from a patient who died in status asthmaticus. Am J Respir Cell Mol Biol. 1995; 13: 748-756.

36. Hoyt RE, Hamilton JF. Ovarian cancer associated with the nephritic syndrome. Obstet Gynaecol. 1987; 70: 513-514.

37. Kim YT, Rha SY, Shim CY, et al. A case of paraneoplastic nephrotic syndrome in a patient with ovarian carcinoma. Yonsei Med J. 2003; 44: 539-543

38. Salazar-Exaire D, Rodriguez A, Galindo-Rujana ME, et al. Membranoproliferative glomerulonephritis associated with a mixed-cell germinal ovary tumour. Am J Nephrol. 2001; 21: 51-54.

39. Beauvais P, Vaudour G, Boccon Gibod L, et al. Membranous nephropathy associated with ovarian tumour in a young girl: recovery after removal. Eur J Pediatr. 1989; 148: 624-625.

40. Couser WG, Wagonfeld JB, Spargo BH, et al. Glomerular deposition of tumour antigen in membranous nephropathy associated with colonic carcinoma. Am J Med. 1974; 57: 962-970. 